\title{
Multistep Optimal Analog-to-Digital Conversion
}

\author{
Daniel E. Quevedo, Student Member, IEEE, and Graham C. Goodwin, Fellow, IEEE
}

\begin{abstract}
An important aspect of analog-to-digital conversion is the impact of quantization errors. This paper outlines how finite horizon constrained optimization methods can be utilized to design converters which minimize a weighted measure of the quantization distortion. We propose a novel converter, which can be implemented as a feedback loop. It embeds $\Sigma \Delta$ conversion in a more general setting and typically provides better performance. We also examine the role played by the associated design parameters in ensuring error convergence.
\end{abstract}

Index Terms-Analog-digital conversion, feedback, optimization, sigma-delta modulation, stability, vector quantization.

\section{INTRODUCTION}

W HEN analog signals are digitized, unavoidably, information loss occurs and the resultant signal will differ from the original, i.e., it will be distorted. In many cases, such as in several digital audio problems [1], it is preferable to minimize the impact of the distortion by pushing its frequency content into appropriate, less harmful, regions.

A widely adopted analog-to-digital converter architecture aimed at addressing these questions is the $\Sigma \Delta$ converter in its various forms, see, e.g., [2]-[4]. Fig. 1 depicts a sampled data diagram of a general (high-order) single-stage $\Sigma \Delta$ converter, as described, e.g., in [3, Ch. 3.2]. In this scheme, the basic quantizer is immersed in a feedback loop which comprises the linear filters $F$ and $G$. A common way of analyzing and designing such a converter is to approximate the quantization errors by white noise which is independent of the signal, see also [5]-[7]. This approximate modeling paradigm leads to a linear quantization model. It motivates one to manipulate the frequency content of the quantization noise present in the digital output of the converter by tuning $F$ and $G .^{1}$

In this paper, we pursue the same general idea of designing an analog-to-digital converter so as to minimize the impact of quantization in different frequency bands. However, we will not follow the standard, approximate, line of reasoning referred to above. Instead, we will pose the problem directly as a weighted $\mathcal{H}_{2}$-optimization problem. By using Parseval's Theorem, we relax the original problem to that of constrained multistep optimization. We show how the solution to this problem can be im-

Manuscript received August 26, 2003; revised May 9, 2004. This paper was recommended by Associate Editor S. Espejo.

The authors are with the School of Electrical Engineering and Computer Science, The University of Newcastle, Newcastle, NSW 2308, Australia (e-mail: dquevedo@ieee.org; eegcg@ee.newcastle.edu.au).

Digital Object Identifier 10.1109/TCSI.2004.843058

${ }^{1}$ In some multibit applications with high order filters and/or dithering this paradigm has proved to be useful. It fails, however, to predict behavior such as the appearance of idle tones and instability, which arise particularly when a single-bit quantizer is used. In these cases, nonlinear techniques become more appropriate, see, e.g., [8].

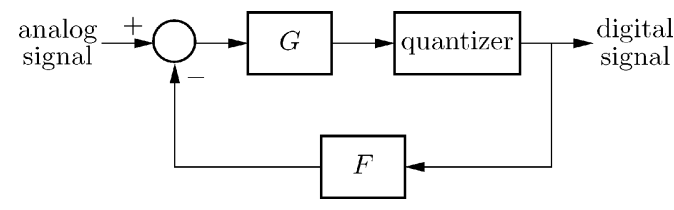

Fig. 1. General (high-order) single-stage $\Sigma \Delta$ converter.

plemented as a feedback loop embodying a new analog-to-digital conversion architecture, which we designate as a multistep optimal converter (MSOC). It encompasses the $\Sigma \Delta$ converter as a special case.

The framework adopted here allows us to re-interpret the general $\Sigma \Delta$ converter as a one-step-optimal solution to the optimization problem considered. The MSOC extends $\Sigma \Delta$-conversion by utilizing multistep optimality and the stability-related concept of error convergence.

This paper builds on earlier work of the current authors documented in [9] and [10]. The novel technical contribution resides in including pre-filtering and final state weighting in the performance measure. These enhancements enable us to:

- incorporate Lyapunov-based stability concepts in the design stage of the analog-to-digital converter;

- embed several $\Sigma \Delta$-converter structures in the proposed MSOC in precise terms;

- develop a multistep optimal extension to the single-loop $\Sigma \Delta$ converter [11].

An outline of the remainder of the paper is as follows: The frequency weighted conversion problem is presented in Section II. In Section III, the MSOC is developed by using a moving horizon concept, which here leads to a multistep quadratic optimization problem. Section IV shows how the MSOC can be implemented as a closed loop. In Section V, we explore, in detail, how to embed several $\Sigma \Delta$ converter structures within this framework. We also develop a multistep extension to the single-loop case. Section VI presents stability properties of the resultant converter architecture. Simulation studies are included in Section VII. Section VIII draws conclusions.

\section{FREQuenCy-Selective ConVERSION}

Consider a scalar analog continuous-time signal $\widetilde{a}_{a}$, defined over $t \in \mathbb{R}^{+}$. The purpose of analog-to-digital conversion is to obtain a discrete-time digital signal $u$. Each value $u(\ell), \ell \in \mathbb{N}$ is restricted to belong to a given finite set of scalars

$$
\mathbb{U}=\left\{s_{1}, \ldots, s_{n_{\cup}}\right\}
$$

where $n_{\cup}$ denotes the cardinality of $\cup$. The scalar $u(\ell)$ approximates, in some well defined sense, the sampled signal $\widetilde{a}$ defined via $\widetilde{a}(\ell) \triangleq \widetilde{a}_{a}[\ell \Delta]$, where $\Delta$ is the sampling period and $\ell \in \mathbb{N}$. 
An immediate solution to the above problem would be to simply approximate each sampled analog value by the nearest element in $\mathbb{U}$, i.e., to set, at sample $\ell=k$

$$
u(k) \longleftarrow q_{\cup}(\widetilde{a}(k))
$$

where $q \cup(\cdot)$ is a quantizer, as defined (in a general vector setting) below.

Definition 1 (Nearest Neighbor Vector Quantizer): Consider a countable set of nonequal vectors, each containing $n_{\mathcal{B}} \in \mathbb{N}$ elements, i.e.,: $\mathcal{B}=\left\{b_{1}, b_{2}, \ldots\right\} \subset \mathbb{R}^{n_{\mathcal{B}}}$.

The nearest neighbor vector quantizer is defined as a mapping $q_{\mathcal{B}}: \mathbb{R}^{n_{\mathcal{B}}} \rightarrow \mathcal{B}$ which assigns to each vector $c \in \mathbb{R}^{n_{\mathcal{B}}}$ the closest element of $\mathcal{B}$ (as measured by the Euclidean norm), i.e., $q_{\mathcal{B}}(c)=$ $b \in \mathcal{B}$ if and only if $b$ satisfies

$$
\|c-b\| \leq\left\|c-b_{i}\right\| \quad \forall b_{i} \in \mathcal{B} .
$$

(A thorough treatment of quantizers and their features can be found in the book [12]. Results from vector quantization theory have also been applied to the analysis of $\Sigma \Delta$ conversion, see [13].)

While the naive decision rule (1) minimizes the overall (unweighted) distortion introduced in the conversion process, this distortion is, in general, highly correlated with the signal $\widetilde{a}$. (It is, indeed, deterministically related.) As a consequence, the spectrum of the distortion can be expected to overlap significantly with that of $\widetilde{a}$. This situation is specially harmful, since it makes it difficult to recover the original analog signal $\widetilde{a}_{a}$ via filtering.

Our goal here is to optimize an appropriate measure of the distortion errors. Specifically, we will aim at an optimal approximation of the pre-filtered signal

$$
a \triangleq H \widetilde{a}
$$

where $H$ is a discrete-time filter. Furthermore, we weight the conversion errors via a stable, causal, linear, time-invariant filter $W$, which can be characterized via ${ }^{2}$

$$
W(\rho)=D+C(\rho I-A)^{-1} B .
$$

This filter can, e.g., represent the typical low-pass filter utilized in oversampled conversion, see, e.g., [14] in order to decimate the converter output. In audio applications it makes sense to choose $W$ as a psycho-acoustic model of the human ear, compare also with work in [9], [15].

We introduce an $\mathcal{H}_{2}$ measure of performance

$$
V \triangleq \frac{1}{2 \pi} \int_{0}^{2 \pi}\left|W\left(e^{j \omega}\right)\left(\mathcal{A}\left(e^{j \omega}\right)-\mathcal{U}\left(e^{j \omega}\right)\right)\right|^{2} d \omega .
$$

In this measure, $W\left(e^{j \omega}\right)$ denotes the frequency response of the filter $W$, while $\mathcal{A}\left(e^{j \omega}\right)$ and $\mathcal{U}\left(e^{j \omega}\right)$ are the (discrete) Fourier transforms of the signals $a$ and $u$, respectively. The cost $V$ penalizes the distortion introduced in the conversion process in a frequency-selective manner.

A converter that minimizes the performance measure $V$ is certainly appealing. The quantized signal $u$ would approximate the filtered input $H \widetilde{a}$ and the distortion, $a-u$, would tend to have

\footnotetext{
${ }^{2}$ Here, and in the remainder of this paper, $\rho$ denotes the forward shift operator, $\rho v(k)=v(k+1)$.
}

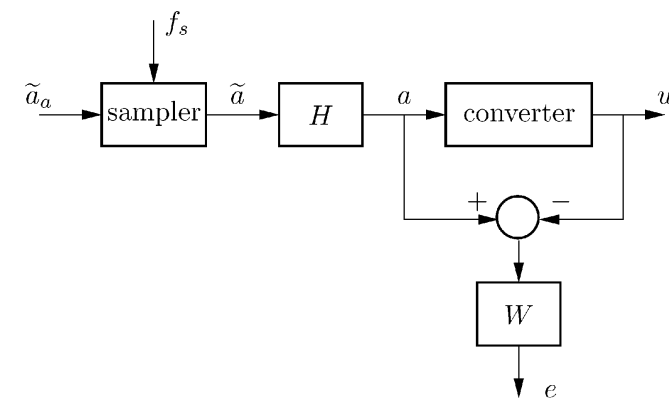

Fig. 2. Weighted analog-to-digital conversion.

a spectrum similar to that of the inverse of the filter $W$. Unfortunately, it is not possible to directly minimize $V$ in practical terms by using expression (5), due to the complexity of solving the associated combinatorial optimization problem. Furthermore, the optimal converter would need to pre-view the entire signal $a$ in order to calculate its Fourier transform. It would therefore be unsuitable for online applications.

In the sequel, we will develop a practical approach to solving the above optimal analog-to-digital conversion problem. The goal is to develop a converter which approximately minimizes the performance measure (5), but is amenable to online applications.

\section{MultisteP OPTIMAL CONVERTER}

In this section, we will utilize the time-domain counterpart of the performance measure given in (5) in order to develop a recursive conversion method, which can be implemented online. For that purpose we will deploy the concept of moving horizon approximation.

\section{A. Time-Domain Formulation}

The quadratic performance measure $V$ can be translated into the time domain by using Parseval's Theorem. This leads to

$$
V=\sum_{\ell=0}^{\infty}(e(\ell))^{2}
$$

where $e(\ell)$ are samples of the filtered conversion distortion $e$, which is the inverse Fourier transform of

$$
E\left(e^{j \omega}\right) \triangleq W\left(e^{j \omega}\right)\left(\mathcal{A}\left(e^{j \omega}\right)-\mathcal{U}\left(e^{j \omega}\right)\right)
$$

and can alternatively be defined via

$$
e \triangleq W(a-u)=W(H \widetilde{a}-u) .
$$

Fig. 2 depicts this approach to measuring the quality of analog-to-digital conversion. In this figure, $f_{s} \triangleq 1 / \Delta$ is the sampling frequency.

Naturally, analog-to-digital conversion based on minimization of $V$ in (6) suffers from identical shortcomings as conversion based on (5) and is therefore unsuitable for practical implementation. This is due to the fact that the value of $V$ depends upon an infinite number of decision variables and also requires that a preview of the entire future input $a$ be available. However, we show below, that we can build on the basic time-domain description (6) to develop a scheme suitable for practical cases. 


\section{B. Finite Horizon Formulation}

In order to develop a practical online conversion scheme, it is necessary to restrict the number of decision variables as well as the number of future values of $a$ utilized in the optimization. Toward that goal, it is convenient to characterize $e$ as the output of the state space system

$$
\begin{aligned}
x(\ell+1) & =A x(\ell)+B(a(\ell)-u(\ell)) \\
e(\ell) & =C x(\ell)+D(a(\ell)-u(\ell)) .
\end{aligned}
$$

This relation follows directly from (4). In (7), $x \in \mathbb{R}^{n}$ is the state vector, and $n \in \mathbb{N}$ is the state dimension, i.e., the order of the filter $W$. Note that, due to the Markovian structure of (7), at time $\ell=k$ the impact of the past trajectories of $a$ and $u$ on future values of $e$ is exactly summarized by means of the present state, $x(k)$.

With this as a background, we next replace the infinite horizon cost function (6) by the following quadratic cost function defined over a finite horizon $N:^{3}$

$$
V_{N} \triangleq\left\|x^{\prime}(k+N)\right\|_{P}^{2}+\sum_{\ell=k}^{k+N-1}\left(e^{\prime}(\ell)\right)^{2}
$$

where $N \in \mathbb{N}$ is fixed and $P$ is a given positive semidefinite matrix.

Starting from the current state value $x(k)$ (see (7)), $V_{N}$ quantifies a measure of the predicted filtered distortion $e$, together with the final state, $x(k+N)$. These predicted quantities are formed based upon the model (7), i.e., they satisfy

$$
\begin{aligned}
x^{\prime}(\ell+1) & =A x^{\prime}(\ell)+B\left(a(\ell)-u^{\prime}(\ell)\right) \\
e^{\prime}(\ell) & =C x^{\prime}(\ell)+D\left(a(\ell)-u^{\prime}(\ell)\right)
\end{aligned}
$$

where $\ell \in\{k, k+1, \ldots, k+N-1\}$ and with initial condition $x^{\prime}(k)=x(k)$.

The finite horizon cost $V_{N}$ proposed in (8) takes into account only a finite number $N$ of constrained values $u$. These decision variables can be grouped into the vector

$$
\vec{u}(k) \triangleq\left[\begin{array}{llll}
u^{\prime}(k) & u^{\prime}(k+1) & \ldots & u^{\prime}(k+N-1)
\end{array}\right]^{T} .
$$

In order to make this dependence explicit, we will write $V_{N}(\vec{u}(k))$. Since the converter output signal is finite-set constrained, so is $\vec{u}(k)$, i.e.,

$$
\vec{u}(k) \in \mathbb{U}^{N}
$$

where $\mathbb{U}^{N} \subset \mathbb{R}^{N}$ is defined via the Cartesian product

$$
\mathbb{U}^{N} \triangleq \mathbb{U} \times \cdots \times \mathbb{U} \text {. }
$$

In the above formulation, only the elements contained in $\vec{u}(k)$ are finite-set constrained. Thus, we will call $N$ the constraint horizon. The value of $N$ fixes the complexity of the calculations needed in order to minimize $V_{N}(\vec{u}(k))$. (Compare with the infinite decision variables in the original cost $V$.) It also reduces the required previewing of $a$ to $N-1$ samples. Since $N$ is left free to the designer, it can be chosen so that the minimization can be carried out online.

${ }^{3}\|x\|_{P}^{2}$ denotes the quadratic form $x^{T} P x$, where $x$ is any vector and $P$ is a matrix

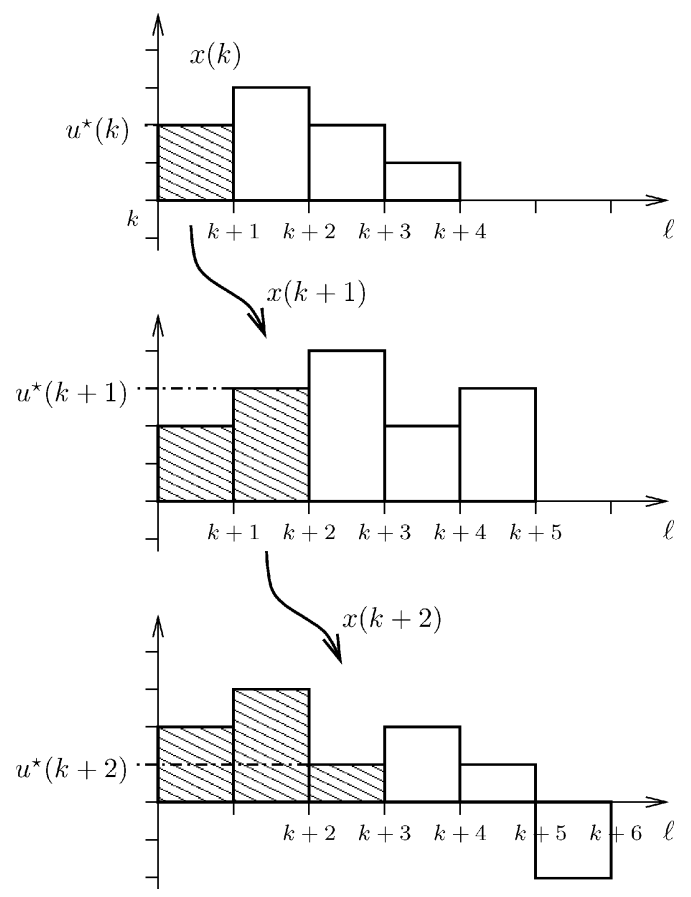

Fig. 3. Moving horizon principle $N=4$.

\section{Moving Horizon Approach}

Minimization of the multistep cost introduced in (8) gives rise to the optimizer

$$
\vec{u}^{\star}(k) \triangleq \arg \min _{\vec{u}(k) \in \mathbb{U}^{N}} V_{N}(\vec{u}(k))
$$

which contains $N$ elements, see (10). Thus, in principle, one could think of an implementation in blocks, where the optimization is carried out every $N$ sampling instants. Unfortunately, the last few elements of $\vec{u}^{\star}(k)$ depend only on a small window of the filtered distortion $e$.

To enhance performance we propose to utilize only the first element of $\vec{u}^{\star}(k)$, namely

$$
u^{\star}(k) \triangleq\left[\begin{array}{llll}
1 & 0 & \ldots & 0
\end{array}\right] \vec{u}^{\star}(k) .
$$

It is this quantity, whose effect on the entire signal $e$ is, in principle, best captured with the $N$ predicted values contained within the horizon examined by the finite horizon cost $V_{N}(\vec{u}(k))$.

The scalar $u^{\star}(k)$ is delivered to the output of the converter, by setting

$$
u(k) \longleftarrow u^{\star}(k)
$$

and is also utilized to update the state according to (7), i.e., we set

$$
x(k+1)=A x(k)+B\left(a(k)-u^{\star}(k)\right) .
$$

At the next sampling instant, this new state value is used to minimize the cost $V_{N}(\vec{u}(k+1))$, yielding $u(k+1)$. This procedure is repeated ad-infinitum. As illustrated in Fig. 3 for the case $N=4$, the constraint horizon of the criterion $V_{N}(\vec{u}(k))$ moves (slides) forward as $k$ increases. The past is propagated 
forward in time via the state sequence $x$, thus, yielding a recursive scheme.

The resultant architecture defines the MSOC, which is of principal interest in the present work. It constitutes an analog-to-digital converter architecture which optimizes the frequency weighted conversion distortion.

It is easy to see that, in general, larger values for $N$ provide better performance, since more data is taken into account in the bit allocation process. In fact, one can expect that, if $N$ is chosen large enough relative to the time scale of $W$, then the effect of $u(k)$ on $e(\ell)$ for $\ell \geq k+N$ will be negligible and the performance of the MSOC will approach that obtained, if the infinite horizon measure of (5) or (6), were to be minimized directly (which, for the reasons explained above, is impractical). This asymptotic behavior is confirmed by simulation studies, see Section VII. Indeed, we will see that modest values of $N$ give near optimal performance, thus rendering the scheme quite easy to implement in practical cases.

In summary, the constraint horizon $N$ allows the designer to trade off performance versus online computational effort and, as will be demonstrated in Section VII, excellent performance can often be achieved with relatively small horizons.

Remark 1 (Relationship to Model Predictive Control): The moving horizon idea advocated above, mirrors the strategy used in model predictive control schemes, see, e.g., [16] or [17, Ch. 23]. Model predictive control has actually been a powerful tool for dealing with constraints in the design of control systems [16]-[20].

\section{IMPLEMENTATION OF MSOC AS FEEDBACK LOOP}

The MSOC described in the previous section requires that one solve the finite-set-constrained quadratic optimization problem (11). In this section, we will develop a solution to this problem, which will allow us to implement the MSOC as a closed loop. Toward this end, it is convenient to define the matrices

$$
\begin{aligned}
& \Gamma \triangleq\left[\begin{array}{c}
C \\
C A \\
\vdots \\
C A^{N-1}
\end{array}\right] \Phi \triangleq\left[\begin{array}{cccc}
w_{0} & 0 & \ldots & 0 \\
w_{1} & w_{0} & \ddots & \vdots \\
\vdots & \ddots & \ddots & 0 \\
w_{N-1} & \ldots & w_{1} & w_{0}
\end{array}\right] \\
& M \triangleq\left[\begin{array}{lllll}
A^{N-1} B & A^{N-2} B & \ldots & A B & B
\end{array}\right] .
\end{aligned}
$$

In the above definition

$$
w_{0} \triangleq D \quad w_{i} \triangleq C A^{i-1} B, \quad i \in\{1,2, \ldots, N-1\}
$$

see (4). Thus, the columns of $\Phi$ are truncated impulse responses of the filter $W$.

The following result gives a dynamical system whose output is the solution to the nonconvex optimization problem (11)-(14).

Theorem 1 (Closed Form Solution): Suppose that $\mathbb{U}^{N}=$ $\left\{v_{1}, v_{2}, \ldots, v_{r}\right\}$, where $r=\left(n_{\cup}\right)^{N}$ and $W$ has realization (4), then the output of the MSOC, see (13), satisfies

$$
u(k)=\left[\begin{array}{llll}
1 & 0 & \ldots & 0
\end{array}\right] \Psi^{-1} q_{\widetilde{\cup} N}(\mathcal{W}(\rho) H(\rho) \widetilde{a}(k)-\mathcal{F}(\rho) u(k)) .
$$

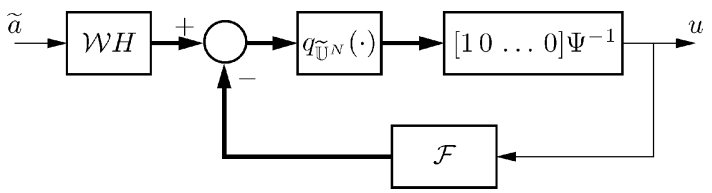

Fig. 4. Implementation of the MSOC as a feedback loop. Thick lines denote vector-signal paths.

In this expression, the transfer functions $\mathcal{W}$ and $\mathcal{F}$ are defined via

$$
\begin{aligned}
& \mathcal{W}(\rho) \triangleq \Psi\left[\begin{array}{llll}
1 & \rho & \ldots & \rho^{N-1}
\end{array}\right]^{T}+\mathcal{F}(\rho) \\
& \mathcal{F}(\rho) \triangleq \Psi^{-T}\left(\Phi^{T} \Gamma+M^{T} P A^{N}\right)(\rho I-A)^{-1} B
\end{aligned}
$$

each having one input and $N$ outputs. The matrix $\Psi$ is square and defined implicitly via

$$
\Psi^{T} \Psi=\Phi^{T} \Phi+M^{T} P M .
$$

The nonlinearity $q_{\widetilde{\cup} N}(\cdot)$ is the nearest neighbor quantizer described in Definition 1. The image of this memoryless mapping is the set

$$
\widetilde{\mathbb{U}}^{N} \triangleq\left\{\widetilde{v}_{1}, \widetilde{v}_{2}, \ldots, \widetilde{v}_{r}\right\} \subset \mathbb{R}^{N}
$$

with

$$
\widetilde{v}_{j}=\Psi v_{j}, \quad v_{j} \in \mathbb{U}^{N}, \quad j \in\{1,2, \ldots, r\} .
$$

Proof: The proof is included in Appendix A.

As a consequence of this theorem, we can implement the MSOC as the feedback loop depicted in Fig. 4, where we have utilized thick lines to denote vector-signal paths.

Note that the complexity of the calculations which need to be performed online is mainly determined by the vector quantizer $q_{\widetilde{U}^{N}}(\cdot)$. It therefore depends upon the cardinality of the search set $\widetilde{U}^{N}$, which is equal to $\left(n_{\cup}\right)^{N}$.

If no final state weighting is used in the cost (8), then, the result stated in Theorem 1 can be simplified, as described in the following corollary.

Corollary 1: With $P=0$, the matrix $\Psi$ in (19) can be chosen according to $\Psi=\Phi$, in which case it follows that

$$
\left[\begin{array}{llll}
1 & 0 & \ldots & 0
\end{array}\right] \Psi^{-1}=\left[\begin{array}{llll}
\frac{1}{w_{0}} & 0 & \ldots & 0
\end{array}\right] .
$$

Furthermore, the transfer function matrices defined in (18) simplify to

$$
\begin{aligned}
\mathcal{W}(\rho) & =\Phi\left[\begin{array}{llll}
1 & \rho & \ldots & \rho^{N-1}
\end{array}\right]^{T}+\mathcal{F}(\rho) \\
\mathcal{F}(\rho) & =\Gamma(\rho I-A)^{-1} B .
\end{aligned}
$$

Note that in this case, $\mathcal{W}$ and $\mathcal{F}$ depend only on the impulse response of $W$ and are therefore independent of the particular realization chosen in (4).

Fig. 4 obviously bears some similarities to the $\Sigma \Delta$ converter depicted earlier in Fig. 1. Indeed, as will be shown in the following section, the MSOC may be regarded as a generalization of the $\Sigma \Delta$ architecture.

\section{RELATIONSHIP TO $\Sigma \Delta$ CONVERSION: HORIZON ONE CASE}

The MSOC has been developed having distortion spectra in mind. Perhaps not surprisingly, it encompasses, as a special 
TABLE I

SPECIFIC FILTER CHOICES

\begin{tabular}{c|c|c}
\hline & $H(\rho)$ & $W(\rho)$ \\
\hline \hline Single-loop & $\rho^{-1}$ & $\frac{\rho}{\rho-1}$ \\
\hline Double-loop & $G g \rho$ & $\frac{\rho^{2}+((B G+b) g-\alpha-\beta) \rho+(\alpha-b g) \beta}{(\rho-\alpha)(\rho-\beta)}$ \\
\hline Multi-loop & 1 & $\left(\frac{\rho}{\rho-1}\right)^{L}$ \\
\hline Bandpass & $\frac{2 r \cos (\theta) \rho-r^{2}}{\rho^{2}-2(1-g) r \cos (\theta) \rho+(1-g) r^{2}}$ & $\frac{\rho^{2}-2(1-g) r \cos (\theta) \rho+(1-g) r^{2}}{\rho^{2}-2 r \cos (\theta) \rho+r^{2}}$ \\
\hline
\end{tabular}

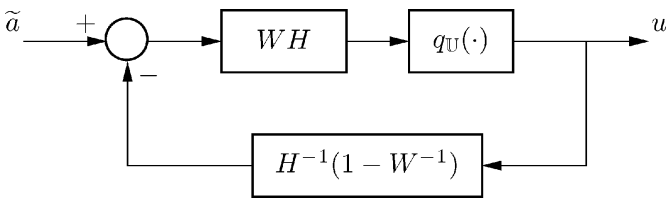

Fig. 5. One-step optimal converter without final state weighting.

case, several $\Sigma \Delta$ converter structures, which also aim to shape the quantization noise. In this section, we will first show how the general high-order single-stage $\Sigma \Delta$ converter can be immersed into the proposed architecture. This insight will allow us to develop, as a special member of the MSOC family, a multistep-optimal extension to the widespread single-loop $\Sigma \Delta$ converter.

\section{A. General Filter}

A particularly simple case of the MSOC is obtained when a unitary constraint horizon with no final state weighting is employed [i.e., $N=1$ and $P=0$ in (8)]. Next, consider a filter $W$ with unitary feed-through, i.e., with $D=1$ in (4). In this case, it follows from (15) and (19) that $\Gamma=C$ and $\Phi=\Psi=1$. Hence, the set $\widetilde{\cup}^{N}$ simplifies to $\mathbb{U}$ and the vector quantizer reduces to a standard scalar quantizer. Equations (17), (21), and (22) then show that the output of the resultant, one-step-optimal analog-to-digital converter satisfies

$$
u(k)=q_{\cup}(W(\rho) H(\rho) \widetilde{a}(k)-(W(\rho)-1) u(k)) .
$$

This special case is depicted in Fig. 5. By comparing this diagram with that of the general (also termed high order) $\Sigma \Delta$ converter presented in Fig. 1, we conclude that the special case of the MSOC described above is equivalent to the latter, if $W$ and $H$ are chosen according to

$$
W=1+F G \quad H=\frac{G}{1+F G} .
$$

Thus, the general single-stage $\Sigma \Delta$ converter of Fig. 1 can be embedded as a special case of the more general frequency weighted analog-to-digital conversion problem. As opposed to existing approaches, see, e.g., [7], we can do this in precise terms, without having to adopt a white-noise model. In particular, we see that the $\Sigma \Delta$-conversion scheme precisely implements the horizon-one solution to (17) as a closed loop.

Different filters $W$ and $H$ allow one to recover specific $\Sigma \Delta$ converter structures. In particular, Table I includes the choices which lead to the standard first-order single-loop $\Sigma \Delta$ converter, ${ }^{4}$ see, e.g., [11], [21]-[24], the second-order double-loop $\Sigma \Delta$ converter of [25] (in [26]-[33] particular cases within that structure are considered), the multiloop architecture described in [34] and bandpass conversion, as described, e.g., in [35]-[37]. (In this table we have characterized the converters using the standard symbols used elsewhere in the literature.) Note that, besides the converters included in Table I, other schemes such as, e.g., the high order $\Sigma \Delta$ converters analyzed in [38], [39] can also be included within our setting.

From the above development, it is apparent that the MSOC captures standard $\Sigma \Delta$-architectures as a special case and also generalizes this paradigm. Indeed, by simply writing the filters $W$ contained in Table I, in the form (4) and choosing $N>1$ we can immediately develop multistep-optimal extensions to these converters. Moreover, in case of the MSOC, a nonzero final state weighting term $\left\|x^{\prime}(k+N)\right\|_{P}^{2}$ can also be chosen, thus giving extra design flexibility.

The main advantage of using a horizon larger than 1 derives from the fact that, with $N>1$, not only the present, but also future values of $\widetilde{a}$ are taken into account in the conversion. Also, choosing $P \neq 0$ may enhance stability properties, see Section VI. As a consequence, and as illustrated in Section VII, the MSOC will in general outperform existing $\Sigma \Delta$ conversion schemes.

Remark 2 (Other vector quantizer converters): It should be emphasized here that the MSOC differs from other converter structures which deploy vector quantizers. In particular, in [40], $\Sigma \Delta$-conversion is generalized to two-input systems, which arise in power electronics applications. The resultant scheme incorporates a hexagonal quantizer, which partitions its two dimensional input space into 7 regions. Alternatively, in [41], the authors propose to implement multibit high-order $\Sigma \Delta$ converters (see Fig. 1), with a vector quantizer. However, as shown above, these cases are only one-step optimal when viewed from the MSOC perspective. In contrast, the latter scheme, as described here, allows for multistep optimality.

\section{B. Single-Loop MSOC}

The first-order single-loop $\Sigma \Delta$ converter included in the first row of Table I deserves special attention. It is commonly char-

\footnotetext{
${ }^{4}$ Although this filter is only marginally stable, we can still deploy our framework by using the cost function (8) directly as a starting point.
} 


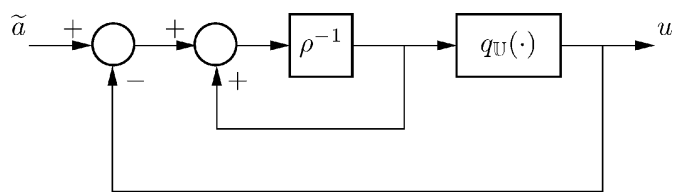

Fig. 6. Single-loop $\Sigma \Delta$ converter.

acterized via the feedback loop depicted in Fig. 6 and has been studied extensively, see, e.g., [11], [21]-[24].5

1) Arbitrary Horizon: In the first-order case, where $W(\rho)=\rho(\rho-1)^{-1}$, one can use the realization (4) with $A=B=C=D=1$. Thus, for arbitrary constraint horizons $N$, the matrices defined in (15) take the form

$$
\Phi=\left[\begin{array}{cccc}
1 & 0 & \ldots & 0 \\
1 & 1 & \ddots & \vdots \\
\vdots & \ddots & \ddots & 0 \\
1 & \ldots & 1 & 1
\end{array}\right], \quad \Gamma=\left[\begin{array}{c}
1 \\
\vdots \\
\vdots \\
1
\end{array}\right]
$$

If we further restrict $P=0$, then, following Corollary 1 , we can set $\Psi=\Phi$ and obtain

$$
\begin{aligned}
& \mathcal{W}(\rho)=\Phi\left[\begin{array}{llll}
1 & \rho & \ldots & \rho^{N-1}
\end{array}\right]^{T}+\frac{1}{\rho-1}\left[\begin{array}{lll}
1 & \ldots & 1
\end{array}\right]^{T} \\
& \mathcal{F}(\rho)=\frac{1}{\rho-1}\left[\begin{array}{lll}
1 & \ldots & 1
\end{array}\right]^{T} .
\end{aligned}
$$

Direct calculation yields

$$
\Phi\left[\begin{array}{c}
1 \\
\rho \\
\rho^{2} \\
\vdots \\
\rho^{N-1}
\end{array}\right]=\left[\begin{array}{c}
1 \\
1+\rho \\
1+\rho+\rho^{2} \\
\vdots \\
1+\rho+\cdots+\rho^{N-1}
\end{array}\right]=\frac{1}{1-\rho}\left[\begin{array}{c}
1-\rho \\
1-\rho^{2} \\
1-\rho^{3} \\
\vdots \\
1-\rho^{N}
\end{array}\right]
$$

and (17) provides

$$
u(k)=\left[\begin{array}{llll}
1 & 0 & \ldots & 0
\end{array}\right] q_{\widetilde{\mathrm{U}}^{N}}(d(k))
$$

with

$$
\begin{aligned}
& d(k) \triangleq \frac{1}{\rho-1} \\
& \cdot\left(\left[\begin{array}{llll}
1 & \rho & \ldots & \rho^{N-1}
\end{array}\right]^{T} \widetilde{a}(k)-\left[\begin{array}{lll}
1 & \ldots & 1
\end{array}\right]^{T} u(k)\right) .
\end{aligned}
$$

The resultant scheme can therefore be implemented as in Fig. 7. It is easy to see that with $N=1$ this scheme reduces to the standard single-loop converter of Fig. 6. Thus, it corresponds to an $N$-step-optimal generalization of the single-loop case. We will call it the single-loop MSOC and note that it is a particular case of the MSOC.

Horizon Two: It is worthwhile exploring the choice $N=2$ in greater detail. In this case, and with $\mathbb{U}=\{-1,1\}$, we obtain that

$$
\begin{aligned}
& \mathbb{U}^{2}=\left\{\left[\begin{array}{l}
-1 \\
-1
\end{array}\right],\left[\begin{array}{c}
-1 \\
1
\end{array}\right],\left[\begin{array}{c}
1 \\
-1
\end{array}\right],\left[\begin{array}{l}
1 \\
1
\end{array}\right]\right\} \\
& \tilde{U}^{2}=\left\{\left[\begin{array}{l}
-1 \\
-2
\end{array}\right],\left[\begin{array}{c}
-1 \\
0
\end{array}\right],\left[\begin{array}{l}
1 \\
0
\end{array}\right],\left[\begin{array}{l}
1 \\
2
\end{array}\right]\right\} .
\end{aligned}
$$

${ }^{5}$ Beyond analog-to-digital conversion, this structure has also been utilized in several other application areas, such as discrete coefficient FIR filter design [42], [43], switch-mode power-supplies [44] and control of solenoid valves for odor blending [45].

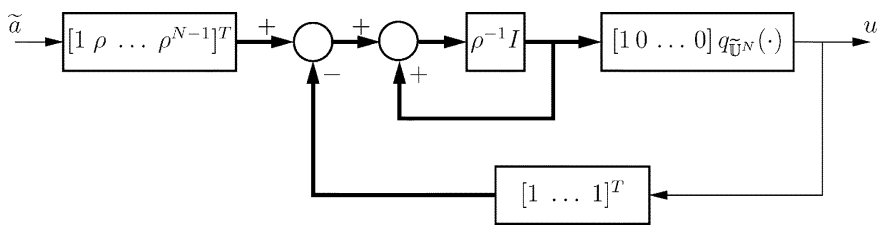

Fig. 7. Single-loop MSOC. Thick lines denote vector-signal paths.

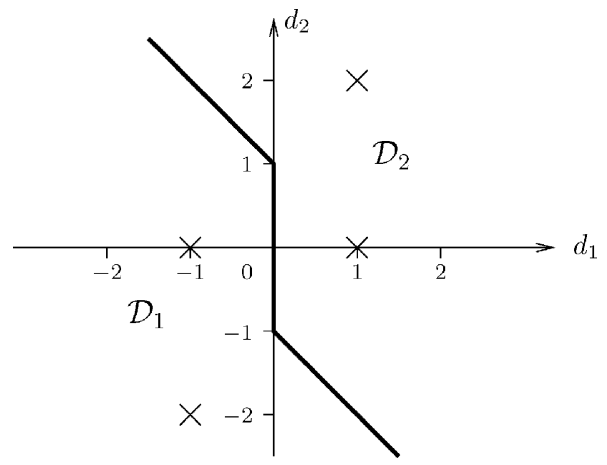

Fig. 8. Partition induced by the quantizer.

The vector quantizer $q_{\widetilde{\cup}^{2}}(\cdot)$ which defines (24) partitions its input space $\mathbb{R}^{2}$ into four regions according to the nearest neighbor rule (2), see, e.g., [12]. Since we are only interested in the first element of $\vec{u}^{\star}(k)$, see (12), only two regions are of significance. These are shown in Fig. 8. Depending upon past converter inputs and outputs, the vector $d(k)$ in (25) will adopt different values. The converter output $u(k)$ is then given by

$$
u(k)= \begin{cases}-1, & \text { if } d(k) \in \mathcal{D}_{1} \\ +1, & \text { if } d(k) \in \mathcal{D}_{2} .\end{cases}
$$

Geometrical arguments allow us to describe the partition of Fig. 8 by means of

$$
\begin{aligned}
u(k)=q_{\cup}\left(q_{\cup}\left(d_{1}(k)+d_{2}(k)-1\right)\right. & \\
& \left.+2\left(q_{\cup}\left(d_{1}(k)\right)+q_{\cup}\left(d_{1}(k)+d_{2}(k)+1\right)\right)\right) .
\end{aligned}
$$

As a consequence, the vector quantizer $q_{\widetilde{\cup}^{2}}(\cdot)$ can be realized with four standard scalar quantizers $q \cup(\cdot)$, which operate on auxiliary scalar signals. The corresponding diagram of the entire two-step optimal single-loop converter can be appreciated in Fig. 9.

Remark 3 (Leakage in the Integrator): In practical applications, it is often the case that the integrator utilized in single-loop $\Sigma \Delta$ converters is not ideal but is affected by leakage and has a nonunity feed-forward gain. As described in [46]-[48], this phenomenon can be modeled by using a first order filter, with gain $g$ and pole located at $0<\alpha<1$. We note that, although these are basically undesired phenomena, their effect can be explicitly taken into account within our framework. In particular, one can choose

$$
H(\rho)=\frac{g}{\rho+g-\alpha} \quad W(\rho)=1+\frac{g}{\rho-\alpha}
$$

and follow similar lines as those described above. (More details on modeling and simulation of nonideal $\Sigma \Delta$-converters can be found, e.g., in [49].) 


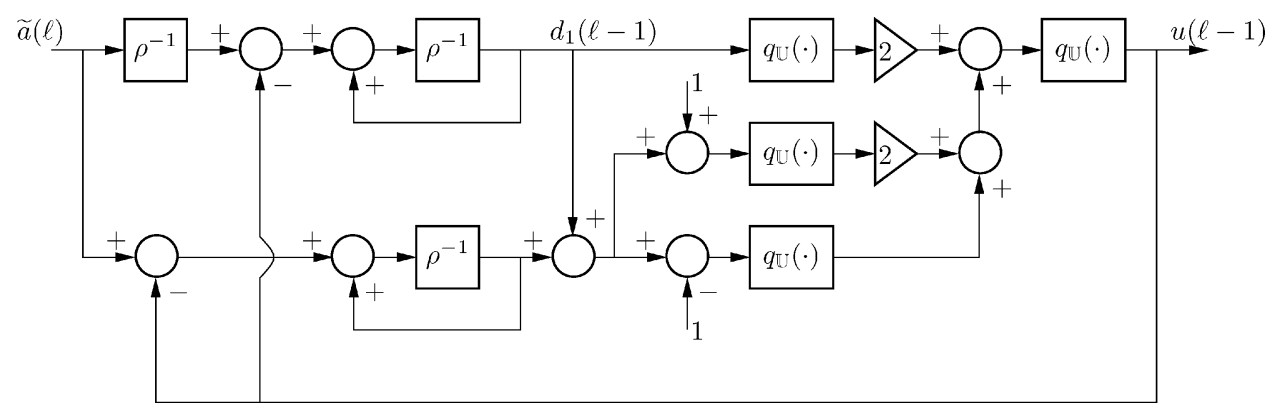

Fig. 9. Diagram of the two-step-optimal single-loop converter.

\section{Achieving Stability Through Final State Weighting}

It is well known that systems, such as the ones described above, incorporating quantization in the feedback loop can exhibit highly nontrivial dynamic behavior. In particular, the case of $\Sigma \Delta$ conversion has been studied extensively, see, e.g., [8], [21], [22], [24], [39], [50]-[53]. It turns out, that the distortion introduced by a $\Sigma \Delta$-converter is deterministically related to the input and depends upon its amplitude and frequency. Even in idle state (i.e., with no input) the output stream may exhibit tones and, in general, it cannot be guaranteed that conversion errors will decay.

One way to overcome this stability problem in $\Sigma \Delta$ conversion resides in utilizing a dithering signal, see, e.g., [3], [6], [54]-[56]. This inclusion of exogenous noise at the input to the nonlinearity makes trajectories behave more random, and less periodic. As a consequence, tone-like behavior can be avoided, but at the expense of a greater noise floor.

For the case of the MSOC considered here, and in light of the relationship established in Section V, one may expect that this scheme will also exhibit complex dynamic behavior. Indeed, the results of [57] and of [58] regarding piecewise-linear mappings can be applied to the MSOC loop of Fig. 4. Inter alia, this predicts the possibility of attractive periodic orbits.

By extending our work related to the moving horizon optimal quantizer described in [9] it is possible to include random dithering in the MSOC, which then can be analyzed following techniques described, e.g., in [59]. Alternatively, and unlike the case of $\Sigma \Delta$ conversion and the moving horizon optimal quantizer, the MSOC can be stabilized without any architectural modifications. To achieve this goal, the final weighting $P$ will turn out to play a pivotal role $^{6}$ as detailed in the following theorem.

Theorem 2 (Error Convergence): Suppose that the signal $a$ is such that it can be eventually quantized, i.e., there exists a finite value $k \in \mathbb{N}$ such that $a(\ell) \in \mathbb{U}, \forall \ell \geq k$. Then, if $P$ is chosen to satisfy the Lyapunov equation

$$
A^{T} P A+C^{T} C=P
$$

then $e(\ell) \rightarrow 0$, as $\ell \rightarrow \infty$.

Proof: The proof is included in Appendix B.

\footnotetext{
${ }^{6}$ The stabilizing effect of final state weighting is known in other contexts, such as, e.g., in Model Predictive Control with saturation-type constraints [18]. However, the choice of $P$ in the finite set constrained case is considerably more difficult and, to the best of our knowledge, has not been addressed, save for our own work in [20]. The latter paper deals with the stability of a related dynamical system when the input is removed. Here, we extend the result to the more practical case of nonzero inputs.
}

It is worth emphasizing here that this result applies to the general case of the MSOC with any (stable) high-order filter $W$ and any finite set $\mathbb{U}$. In particular, if $P$ is chosen as a solution to (26) and $0 \in \mathbb{U}$, then the output of any member of the MSOC-family exhibits no idle tones. This is in stark contrast to the bulk of the work available in the existing literature, see, e.g., [8], [28]-[30], [32], [35], [37], [60], [61], which aims at establishing stability related concepts only for specific $\Sigma \Delta$ converter choices.

Remark 4 (The Horizon One Case): An interesting application of Theorem 2 is related to the horizon one case. Following the relationship established in detail in Section V, general high-order single-stage $\Sigma \Delta$ converters can be stabilized by using final state weighting following (26). Note that, in this case where $N=1$, the vector quantizer of the MSOC reduces to a scalar quantizer so that the stabilization is achieved at the expense of only reduced increase in online computational effort/circuit complexity.

Although, strictly speaking, Theorem 2 is of significance only for input signals which give rise to an eventually quantized sequence $a$, simulation studies indicate that setting $P$ as the positive definite solution to the Lyapunov (26) is, in general, a good choice. As shown in Section VII-B below, it helps to avoid limit cycles and tonal distortion. Thus, the MSOC provided with the stabilizing matrix $P$ given in (26) can be regarded as a deterministically dithered converter. Unlike other such converter architectures, see, e.g., the bit-flipping technique of [62], [63], the adaptive integrator-output bounding converter [64] or the stabilizing $\Sigma \Delta$-conversion scheme of [65], the MSOC is a nonadaptive time-invariant structure, and is, therefore, easier to implement.

Remark 5 (Infinite Horizons): The choice for the final state weighting matrix suggested by Theorem 2 can be related to the infinite horizon measure $V$ in (6) by using standard results related to Lyapunov stability theory, see, e.g., [66]. More precisely, it follows that, if $u^{\prime}(\ell)$ is equal to $a(\ell)$, for every $\ell \geq$ $k+N$ and $P$ satisfies (26), then

$$
\left\|x^{\prime}(k+N)\right\|_{P}^{2}=\sum_{\ell=k+N}^{\infty}\left(e^{\prime}(\ell)\right)^{2}
$$

where the terms in the sum are subject to the filter dynamics as in (9), starting at $x^{\prime}(k+N)$.

Despite the fact that, in general, the finite set constraints preclude $u^{\prime}(\ell)$ to be equal to $a(\ell)$ so that (27) can only hold approximately, this expression allows one to interpret Theorem 2 as a stability result based upon infinite horizon optimality. Viewed 
from that angle, one can think of the term $\left\|x^{\prime}(k+N)\right\|_{P}^{2}$ as a mechanism for summarizing the infinite horizon behavior.

\section{Simulation RESUlts}

In order to illustrate characteristics of various members of the MSOC family, we will measure performance using an input signal $\widetilde{a}(\ell)$ defined over $\ell=1,2, \ldots, T_{f}$ via the sample variance of the distortion defined as

$$
S \triangleq \frac{1}{T_{f}} \sum_{\ell=1}^{T_{f}}(H(\rho) \widetilde{a}(\ell)-u(\ell))^{2}
$$

and by means of the sample variance of the filtered distortion

$$
S_{f} \triangleq \frac{1}{T_{f}} \sum_{\ell=1}^{T_{f}}(e(\ell))^{2}
$$

value, which is related to the cost (6). Both measures will be evaluated with data stemming from time-domain simulations.

\section{A. Single-Loop MSOC}

In what follows, we will analyze the behavior of the Single-loop MSOC described in Section V-B (see Fig. 7) with the binary alphabet $U=\{-1,1\}$.

1) Periodic Inputs: We first utilize a periodic input signal of the form

$$
\begin{aligned}
& \tilde{a}(\ell)=0.1 \pi^{-1}+A \sin (2 \pi f \ell), \\
& \quad \ell=1,2, \ldots, T_{f}, \quad T_{f}=5000
\end{aligned}
$$

which corresponds to a sampled sinusoid with a small offset ${ }^{7}$. Its amplitude is $A$ and its frequency is equal to $f$ times the sampling frequency $f_{s}$. Thus, $f$ denotes relative frequency. If the Nyquist criterion is to be satisfied, then $f$ should lie in the range $0 \leq$ $f<0.5$. Having in mind oversampling applications, we restrict $f$ further.

Fig. 10 illustrates the effect of input frequency $f$ for a fixed amplitude, $A=2$. It shows the values of $S$ defined in (28) for the case of Single-loop $\Sigma \Delta$-conversion as in Fig. 6 and for the Single-loop MSOC of Fig. 7 with horizons $N=2$ and $N=3$. It can be seen in the figure that the MSOC introduces less distortion than the $\Sigma \Delta$ converter. Moreover, choosing $N=$ 3 outperforms the smaller constraint horizon, $N=2$. These results are confirmed in Fig. 11, which shows the effect of input amplitude $A$. Again, the MSOC schemes outperform the $\Sigma \Delta$ converter, although the effect becomes significant only at larger amplitudes.

2) Random Inputs: Next, we examine the case of a random input. For that purpose, we choose $\widetilde{a}$ to be a white noise process, where each observation $\widetilde{a}(\ell), \ell=1, \ldots, T_{f}=10^{4}$, where $T_{f}=$ $10^{4}$ is uniformly distributed in the interval $[-2,2]$.

Typical power spectral densities of the resultant (unfiltered) distortion, $a-u$, for the Single-loop $\Sigma \Delta$ converter and the

\footnotetext{
${ }^{7}$ Following the results of [21], [67] (see also [31]), we included an irrational dc-component in order to avoid singular behavior, which especially in the single-loop $\Sigma \Delta$ converter case, would deteriorate performance significantly. For the same reason, unless otherwise stated, in all simulations, $f$ is chosen to be irrational.
}

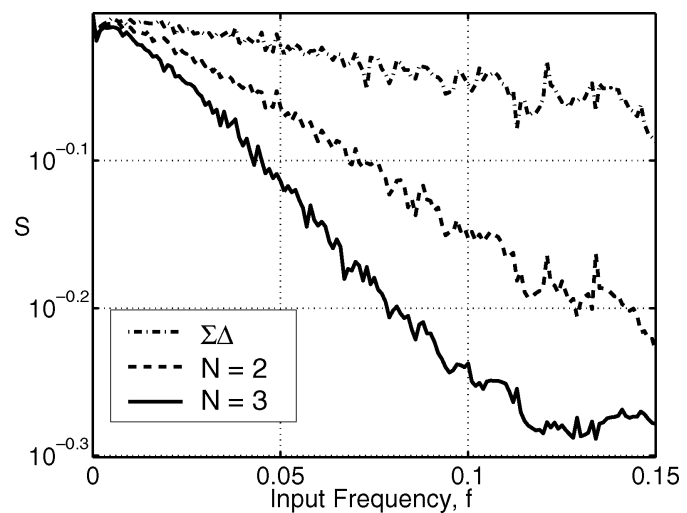

Fig. 10. Effect of $f$, single-loop $\Sigma \Delta$ conversion and single-loop MSOC with $N=2,3(A=2)$.

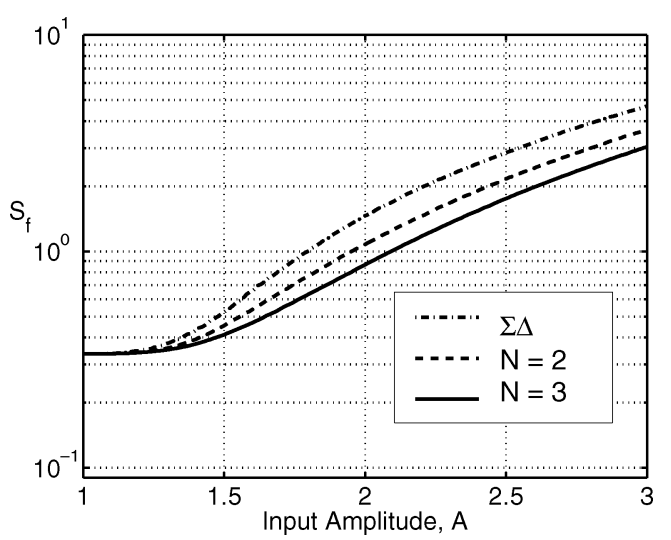

Fig. 11. Effect of $A$, single-loop $\Sigma \Delta$ conversion and single-loop MSOC with $N=2,3\left(f=7 e^{-4} \approx 0.128\right)$.

Single-loop MSOC with $N=10$ are included in Fig. 12. As might have been anticipated, while both schemes shape the quantization effect, the overall distortion level is lower in case of the Single-loop MSOC. The performance improvement is, in this case, typically circa $4 \mathrm{~dB}$ in the relevant frequency band. This is further documented in Fig. 13 which illustrates the effect of the constraint horizon $N$ on performance, as measured by the filtered variance $S_{f}$ defined in (29). It shows that the performance of the single-loop MSOC is monotonic and asymptotic in $N$. (The effect of $N$ on $S$ is similar.)

\section{B. Multistep Optimal Converter}

A more general case results when using the 3-bit alphabet $\mathbb{U}=\{-4,-3, \ldots, 3\}$ and the filters

$$
H(\rho)=1 \quad W(\rho)=\frac{\rho+1.6}{\rho+0.3} .
$$

This filter $W$ is low pass, stable, and nonminimum phase. The solution to the associated Lyapunov equation (26) of Theorem 2 is, in this case, $P \approx 1.8571$. In the sequel, we will denote it as the stabilizing $P$.

1) DC Inputs: We first choose $\widetilde{a}$ to be a dc input $\left(T_{f}=\right.$ 5000) and simulate three cases: the general $\Sigma \Delta$ converter of Fig. 1 with $G$ and $F$ chosen according to (31) and (23), the MSOC with $N=2$ and $P=0$, and the MSOC with $N=1$ and with the stabilizing $P$. 


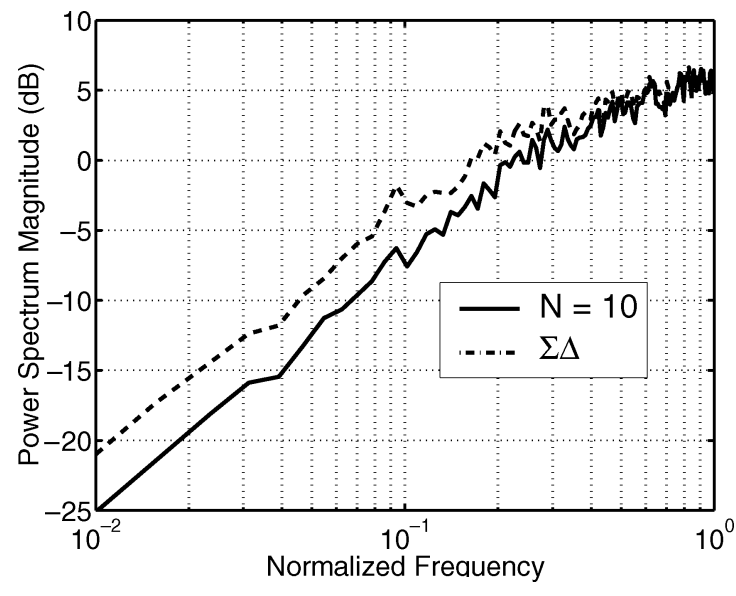

Fig. 12. Distortion spectra in the single-loop case with random input.

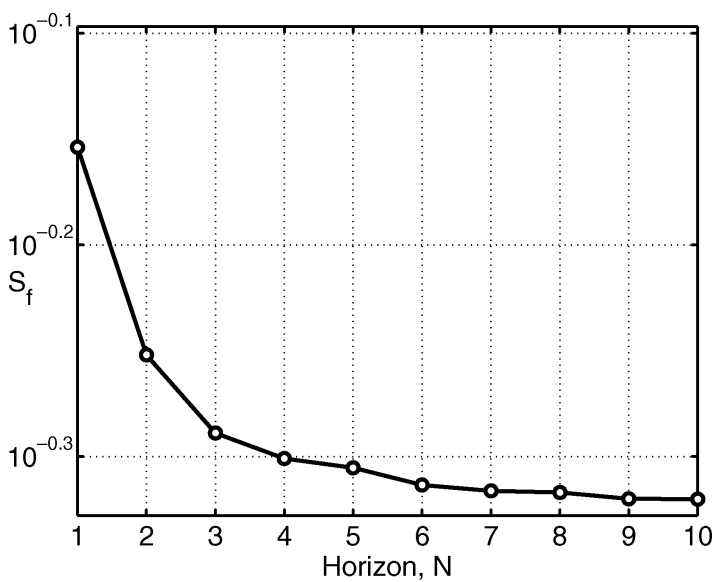

Fig. 13. Effect of $N$ on $S_{f}$ for the single-loop case with random input.

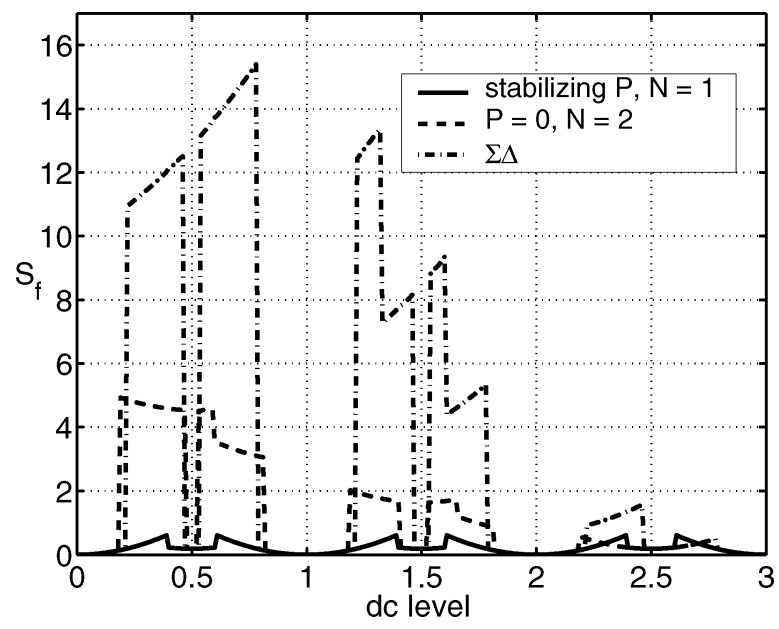

Fig. 14. Effect of dc level on $S_{f}$.

Fig. 14 illustrates the results. As can be seen, the effect of the final state weighting matrix $P$ is remarkable. Although the result of Theorem 2 is useful here, in principle, only for the integer valued dc levels, the MSOC with stabilizing $P$ far outperforms the other two converters. The main reason for this, is that in case of the $\Sigma \Delta$ converter and, to a lesser degree also for the MSOC with $P=0$, the output stream converges for most input streams to periodic sequences comprising mainly the extremal values

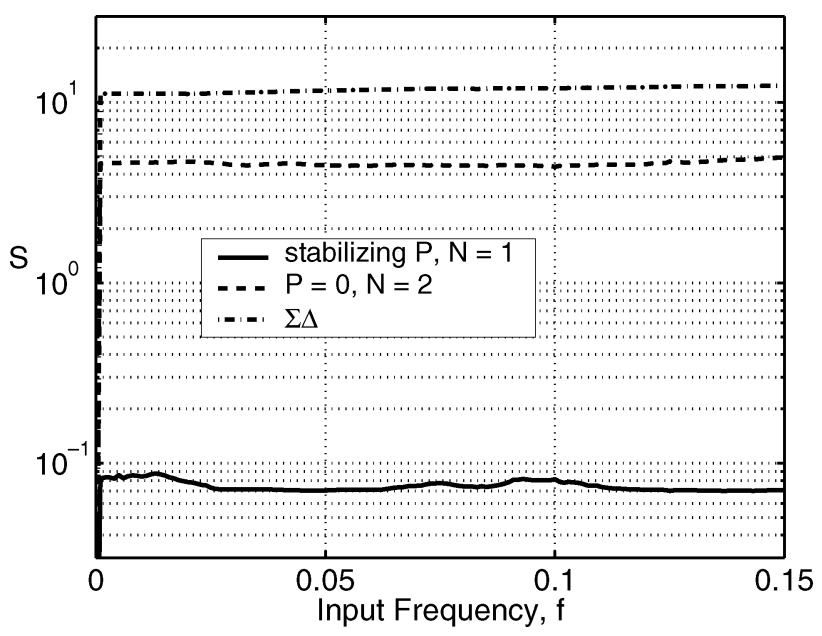

Fig. 15. Effect of $f$ on $S(A=2)$.

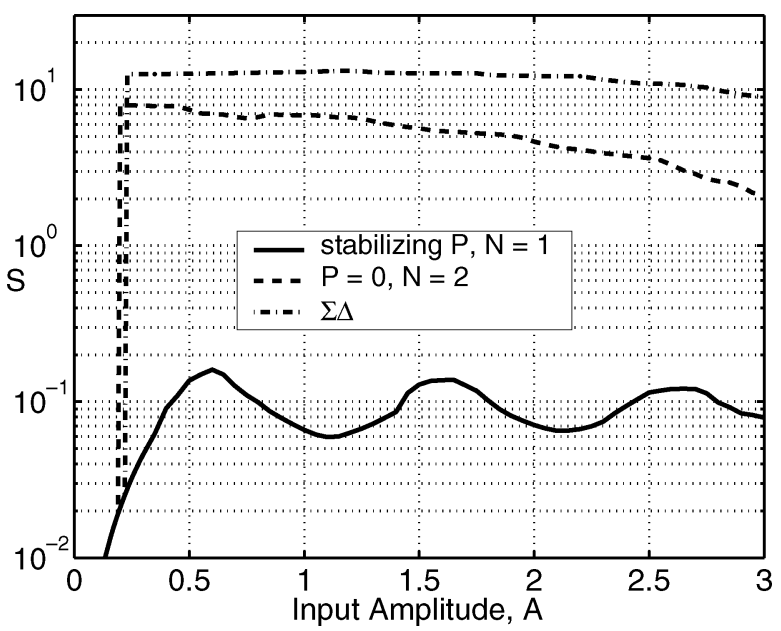

Fig. 16. Effect of $A$ on $S\left(f=7 e^{-4} \approx 0.128\right)$.

-4 and 3 . This phenomenon is avoided by choosing the stabilizing $P$ and leads to significant performance enhancement.

It should be emphasized here that, in this case, the MSOC with stabilizing $P$ uses only a scalar quantizer, since a unitary constraint horizon $N=1$ was chosen, see also Remark 4 . Thus, the improvement in performance in comparison with $\Sigma \Delta$ conversion is obtained with negligible increase in online complexity.

2) Periodic Inputs: Similar results can be observed for other input signals. In particular, Figs. 15 and 16 visualize the performance achieved in the three cases analyzed above when sinusoidal signals, as in (30) are applied to the input. As can be seen, the stabilizing effect of $P$ yields performance improvement which is typically more than $20 \mathrm{~dB}$.

Further insight can also be gained by inspecting the signal spectra. Fig. 17 is obtained by choosing a sinusoidal input as in (30) with $A=2$ and $f=1 / 7$. As can be seen, the $\Sigma \Delta$ converter gives rise to a signal with many (direct and shifted) harmonics (compare to the analytical results of [68]). In particular, it contains a period-two component, which dominates the 


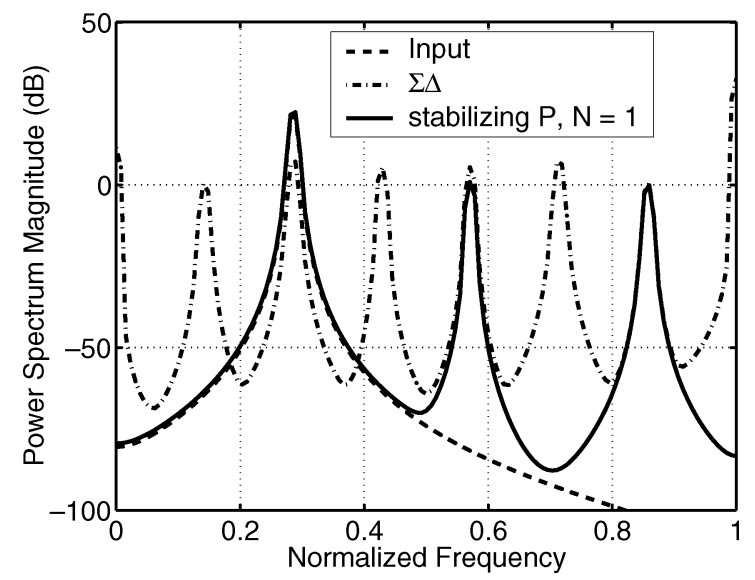

Fig. 17. Spectra of periodic input and converted signals.

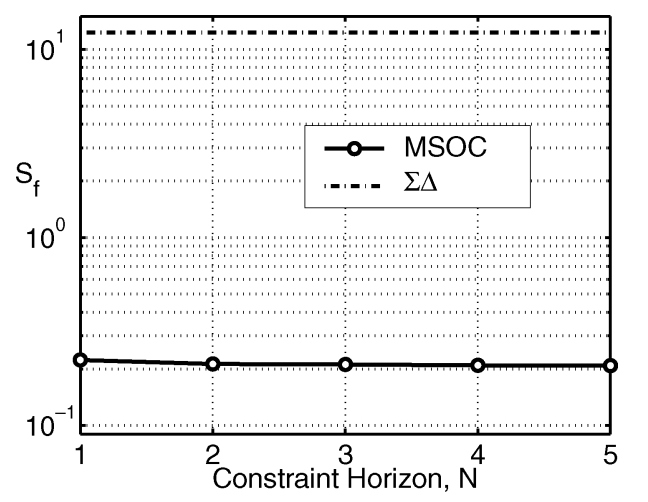

Fig. 18. Performance of the stabilizing MSOC and $\Sigma \Delta$ conversion (random input).

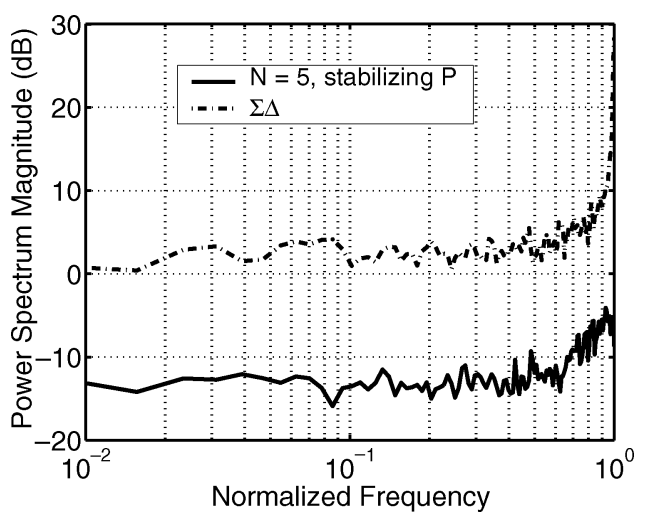

Fig. 19. Distortion spectra (random input).

input-frequency component by more than $20 \mathrm{~dB} .{ }^{8}$ In comparison, the MSOC with unitary horizon and $P$ set to the stabilizing value yields an output signal whose most significant contribution is at the input frequency.

3) Random Inputs: Finally, we choose the signal $\widetilde{a}$, to consist of 4000 observations of an independent random variable, uniformly distributed over the interval $[-4.5,3.5]$. Fig. 18 shows the performance achieved with $\Sigma \Delta$ conversion and for

\footnotetext{
${ }^{8}$ Note that these high-frequency tones are specially dangerous, since they may couple easily into the baseband due to clock noise.
}

the MSOC with stabilizing $P$ and different constraint horizons. Again, the MSOC clearly outperforms the general $\Sigma \Delta$ converter. It is also interesting to note that, in this case, a small horizon of $N=2$ gives near optimal performance, compare to Fig. 13. As depicted in Fig. 19, the power spectrum density of the distortion caused by $\Sigma \Delta$-conversion has a large component at the Nyquist frequency. This is due to an instability of the loop, which manifests itself as intermittent limit cycles (short term periodicities), which comprise only extremal values of $\mathbb{U}$ and lead to performance degradation. The stabilizing choice for $P$ given by the Lyapunov equation (26) suppresses this phenomenon.

\section{CONCLUSION}

In this paper, we have presented a novel analog-to-digital conversion methodology, which is designed by minimizing a measure of the frequency-weighted distortion. Based upon moving horizon optimization ideas, the proposed MSOC can be implemented as a feedback loop, comprising two linear filters and a quantizer. The resultant architecture is amenable to online applications.

Furthermore, we have shown that $\Sigma \Delta$-conversion corresponds to a particular case of the new MSOC-scheme. In particular, various $\Sigma \Delta$-converters proposed in the literature have been shown to correspond to the one-step-optimal form of the multistep optimization problem examined here.

The MSOC has two prime advantages. Firstly, it allows for multistep optimality. Secondly, final state weighting can be used to ensure stability-like properties and, thus, to avoid tone-like behavior without requiring the use of random dithering. As apparent from the simulation studies included, both aspects allow for enhanced performance.

\section{APPENDIX A \\ PROOF OF THEOREM 1}

By iterating (7), one can see that the cost function $V_{N}(\vec{u}(k))$ can be written in vector form as

$$
\begin{aligned}
V_{N}(\vec{u}(k)) & =(\Phi(\vec{a}(k)-\vec{u}(k))+\Gamma x(k))^{T}(\Phi(\vec{a}(k)-\vec{u}(k)) \\
& +\Gamma x(k))+\left\|M(\vec{a}(k)-\vec{u}(k))+A^{N} x(k)\right\|_{P}^{2}
\end{aligned}
$$

where the matrices $M, \Phi$ and $\Gamma$ are defined in (16) and (19), while

$$
\vec{a}(k) \triangleq\left[\begin{array}{llll}
a(k) & a(k+1) \quad \ldots \quad a(k+N-1)
\end{array}\right]^{T} .
$$

Direct manipulation of (32) yields

$$
\begin{aligned}
V_{N}(\vec{u}(k))= & \|\vec{u}(k)\|_{\Psi^{T} \Psi}^{2}-2(\vec{u}(k))^{T}\left(\left(\Psi^{T} \Psi\right) \vec{a}(k)\right. \\
& \left.+\left(\Phi^{T} \Gamma+M^{T} P A^{N}\right) x(k)\right)+\|x(k)\|_{\Gamma^{T} \Gamma+\left(A^{N}\right)^{T} P A^{N}}^{2} \\
& +2(\vec{a}(k))^{T}\left(\Phi^{T} \Gamma+M^{T} P A^{N}\right) x(k)+\|\vec{a}(k)\|_{\Psi^{T} \Psi}^{2}
\end{aligned}
$$

Note that only the first two terms on the right-hand side of (34) depend upon the decision variable $\vec{u}(k)$. 
We introduce the change of variables, $\vec{\mu}(k)=\Psi \vec{u}(k)$. This transforms $\mathbb{U}^{N}$ into $\widetilde{\mathbb{U}}^{N}$ defined in (20). Equation (34) then allows us to rewrite the optimizer (11) as

$$
\vec{u}^{\star}(k)=\Psi^{-1} \arg \min _{\vec{\mu}(k) \in \widetilde{\cup}^{N}} J_{N}(\vec{\mu}(k))
$$

where

$$
\begin{aligned}
J_{N}(\vec{\mu}(k)) & \triangleq(\vec{\mu}(k))^{T} \vec{\mu}(k) \\
& -2(\vec{\mu}(k))^{T}\left(\Psi \vec{a}(k)+\Psi^{-T}\left(\Phi^{T} \Gamma+M^{T} P A^{N}\right) x(k)\right) .
\end{aligned}
$$

The level sets of $J_{N}$ are spheres in $\mathbb{R}^{N}$, centred at $\Psi \vec{a}(k)+$ $\Psi^{-T}\left(\Phi^{T} \Gamma+M^{T} P A^{N}\right) x(k)$. Hence, the constrained optimizer is given by the nearest neighbor

$$
\begin{aligned}
\arg \min _{\vec{\mu}(k) \in \widetilde{\cup}^{N}} & J_{N}(\vec{\mu}(k)) \\
& =q_{\widetilde{U}^{N}}\left(\Psi \vec{a}(k)+\Psi^{-T}\left(\Phi^{T} \Gamma+M^{T} P A^{N}\right) x(k)\right) .
\end{aligned}
$$

Equations (35) and (12) then yield

$$
\begin{aligned}
u(k)= & {\left[\begin{array}{llll}
1 & 0 & \ldots & 0
\end{array}\right] \Psi^{-1} } \\
& \cdot q_{\widetilde{\cup}^{N}}\left(\Psi \vec{a}(k)+\Psi^{-T}\left(\Phi^{T} \Gamma+M^{T} P A^{N}\right) x(k)\right) .
\end{aligned}
$$

It follows directly from (3), (14) and (33) that

$$
\begin{aligned}
\vec{a}(k) & =\left[\begin{array}{llll}
1 & \rho & \ldots & \rho^{N-1}
\end{array}\right]^{T} H(\rho) \widetilde{a}(k) \\
x(k) & =(\rho I-A)^{-1} B(H(\rho) \widetilde{a}(k)-u(k)) .
\end{aligned}
$$

The result (17) follows by substituting these expressions into (36).

\section{APPENDIX B \\ PROOF OF THEOREM 2}

The proof is based upon Lyapunov stability [69] and utilizes the sequence of optimal costs defined as

$$
V_{N}^{\star}(\ell) \triangleq V_{N}\left(\vec{u}^{\star}(\ell)\right), \quad \ell \in \mathbb{N} .
$$

Suppose that an optimal sequence $\vec{u}^{\star}(k)$ has been found, where

$$
\vec{u}^{\star}(k)=\left[\begin{array}{llll}
u_{k} & u_{k+1} & \ldots & u_{k+N-1}
\end{array}\right]^{T} .
$$

Next, at sample $\ell=k+1$, consider a related sequence

$$
u^{s}=\left[\begin{array}{llll}
u_{k+1} & u_{k+2} \ldots & u_{k+N-1} & a(k+N)
\end{array}\right]^{T}
$$

which belongs to $\bigcup^{N}$. Due to optimality, it follows that $V_{N}^{\star}(k+$ 1) $\leq V_{N}\left(u^{s}\right)$. Direct calculation then yields

$$
\begin{aligned}
V_{N}\left(u^{s}\right)= & V_{N}^{\star}(k)+\|A x(k+N)\|_{P}^{2}-\|x(k+N)\|_{P}^{2} \\
& +(e(k+N))^{2}-(e(k))^{2} \\
= & V_{N}^{\star}(k)+\|A x(k+N)\|_{P}^{2}-\|x(k+N)\|_{P}^{2} \\
& +(C x(k+N))^{2}-(e(k))^{2}
\end{aligned}
$$

since, with $u^{s}$ given by (37), predictions of $e$ used in $V_{N}^{\star}(k)$ and in $V_{N}\left(u^{s}\right)$ coincide. Hence

$$
\begin{array}{r}
V_{N}^{\star}(k+1)-V_{N}^{\star}(k) \leq\|x(k+N)\|_{A^{T} P A-P+C^{T} C}^{2}-(e(k))^{2} \\
=-(e(k))^{2} \leq 0
\end{array}
$$

after replacing (26) and the sequence $V_{N}^{\star}(k)$. As a consequence, $\lim _{\ell \rightarrow \infty} V_{N}^{\star}(\ell)$ exists and $V_{N}^{\star}(\ell+1)-V_{N}^{\star}(\ell) \rightarrow 0$. Due to (38), also $e(\ell) \rightarrow 0$, which completes the proof.

\section{ACKNOWLEDGMENT}

The authors gratefully acknowledge helpful suggestions made by the reviewers of the original version of this paper.

\section{REFERENCES}

[1] K. C. Pohlmann, Principles of Digital Audio, 4th ed. New York: McGraw-Hill, 2000

[2] Oversampling Delta-Sigma Data Converters Theory, Design and Simulation, J. C. Candy and G. C. Temes, Eds., IEEE Press, New York, 1992.

[3] Delta-Sigma Data Converters: Theory, Design and Simulation, S. R. Norsworthy, R. Schreier, and G. C. Temes, Eds., IEEE Press, Piscataway, NJ, 1997.

[4] P. M. Aziz, H. V. Sorensen, and J. V. Spiegel, "An overview of sigma-delta converters," IEEE Signal Processing Mag., vol. 13, no. 1, pp. 61-84, Jan. 1996.

[5] S. K. Tewksbury and R. W. Hallock, "Oversampled, linear predictive and noise-shaping coders of order $N>1$," IEEE Trans. Circuits Syst., vol. CAS-25, pp. 436-447, Jul. 1978.

[6] S. P. Lipschitz, R. A. Wannamaker, and J. Vanderkooy, "Quantization and dither: A theoretical survey," J. Audio Eng. Soc., vol. 40, no. 5, pp. 355-375, 1992.

[7] R. Schreier, "An empirical study of high-order single bit delta-sigma modulators," IEEE Trans. Circuits Syst. II, Analog Digit. Signal Process., vol. 40, no. 8, pp. 461-466, Aug. 1993.

[8] O. Feely, "A tutorial introduction to nonlinear dynamics and chaos and their application to sigma-delta modulators," Int. J. Circuit Theory Appl., vol. 25, pp. 347-367, 1997.

[9] G. C. Goodwin, D. E. Quevedo, and D. McGrath, "Moving-horizon optimal quantizer for audio signals," J. Audio Eng. Soc., vol. 51, no. 3, pp 138-149, 2003.

[10] D. E. Quevedo and G. C. Goodwin, "Audio quantization from a receding horizon control perspective," in Proc. Amer. Contr. Conf., 2003, pp. 4131-4136.

[11] R. M. Gray, "Oversampled sigma-delta modulation," IEEE Trans. Commun., vol. COM-35, no. 5, pp. 481-489, May 1987.

[12] A. Gersho and R. M. Gray, Vector Quantization and Signal Compression. Norwell, MA: Kluwer, 1992.

[13] N. T. Thao, "Vector quantization analysis of $\Sigma \Delta$ modulation," IEEE Trans. Signal Processing, vol. 44, no. 4, pp. 808-817, Apr. 1996.

[14] L. Lo Presti, "Efficient modified-sinc filters for sigma-delta A/D converters," IEEE Trans. Circuits Syst. II, Analog Digit. Signal Process., vol. 47, no. 11, pp. 1204-1213, Nov. 2000.

[15] C. Dunn and M. Sandler, "Psychoacoustically optimal sigma-delta modulation," J. Audio Eng. Soc., vol. 45, no. 4, pp. 212-223, 1997.

[16] J. M. Maciejowski, Predictive Control with Constraints. Englewood Cliffs, NJ: Prentice-Hall, 2002.

[17] G. C. Goodwin, S. F. Graebe, and M. E. Salgado, Control System Design. Englewood Cliffs, NJ: Prentice-Hall, 2001.

[18] D. Q. Mayne, J. B. Rawlings, C. V. Rao, and P. O. M. Scokaert, "Constrained model predictive control: Optimality and stability," Automatica, vol. 36, no. 6, pp. 789-814, 2000. 
[19] S. J. Qin and T. A. Badgwell, "A survey of industrial model predictive control technology," Contr. Eng. Pract., vol. 11, pp. 733-764, 2003.

[20] D. E. Quevedo, G. C. Goodwin, and J. A. De Doná, "Finite constraint set receding horizon control," Int. J. Robust Nonlin. Contr., vol. 14, no. 4, pp. 355-377, 2004.

[21] R. M. Gray, W. Chou, and P. W. Wong, "Quantization noise in single-loop sigma-delta modulation with sinusoidal inputs," IEEE Trans. Commun., vol. 37, no. 9, pp. 956-968, Sep. 1989.

[22] P. W. Wong and R. M. Gray, "Sigma-delta modulation with I.I.D. Gaussian inputs," IEEE Trans. Inform. Theory, vol. 36, no. 4, pp. 784-798, Jul. 1990.

[23] O. Feely and L. O. Chua, "Nonlinear dynamics of a class of analog-todigital converters," Int. J. Bifurc. Chaos, vol. 2, no. 2, pp. 325-340, 1992.

[24] T. Koski, "Statistics of the binary quantizer error in single-loop sigma-delta modulation with white Gaussian input," IEEE Trans. Inform. Theory, vol. 41, pp. 931-943, Jul. 1995.

[25] S. Hein, "Exploiting chaos to suppress spurious tones in general double-loop $\Sigma \Delta$ modulators," IEEE Trans. Circuits Syst. II, Analog Digit. Signal Process., vol. 40, no. 10, pp. 651-659, Oct. 1993.

[26] J. C. Candy, "A use of double integration in sigma-delta modulation," IEEE Trans. Commun., vol. COM-33, no. 3, pp. 249-258, Mar. 1985.

[27] V. Friedman, "The structure of the limit cycles in sigma-delta modulation," IEEE Trans. Commun., vol. 36, no. 8, pp. 972-979, Aug. 1988.

[28] H. Wang, "A geometric view of $\Sigma \Delta$ modulations," IEEE Trans. Circuits Syst. II, Analog Digit. Signal Process., vol. 39, no. 6, pp. 402-405, Jun. 1992.

[29] S. C. Pinault and P. V. Lopresti, "On the behavior of the double-loop sigma-delta modulator," IEEE Trans. Circuits Syst. II, Analog Digit. Signal Process., vol. 40, no. 8, pp. 467-479, Aug. 1993.

[30] S. Hein and A. Zakhor, "On the stability of sigma-delta modulators," IEEE Trans. Signal Processing, vol. 41, no. 7, pp. 2322-2348, Jul. 1993.

[31] S. Rangan and B. Leung, "Quantization noise spectrum of double-loop sigma-delta converter with sinusoidal input," IEEE Trans. Circuits Syst. II, Analog Digit. Signal Process., vol. 41, no. 2, pp. 168-173, Feb. 1994.

[32] R. Farrell and O. Feely, "Bounding the integrator outputs of secondorder sigma-delta modulators," IEEE Trans. Circuits Syst. II, Analog Digit. Signal Process., vol. 45, no. 6, pp. 691-702, Jun. 1998.

[33] D. Fournier-Prunaret, O. Feely, and I. Taralova-Roux, "Lowpass sigma-delta modulation: An analysis by means of the critical lines tool," Nonlin. Anal., vol. 47, pp. 5343-5355, 2001.

[34] N. He, F. Kuhlmann, and A. Buzo, "Multiloop sigma-delta quantization," IEEE Trans. Inform. Theory, vol. 38, no. 3, pp. 1015-1028, May 1992.

[35] O. Feely and D. Fitzgerald, "Bandpass sigma-delta modulation - An analysis from the perspective of nonlinear dynamics," in Proc. IEEE Int. Symp. Circuits and Systems, vol. 3, Atlanta, GA, 1996, pp. 146-149.

[36] A. C. Davies, "Periodic nonlinear oscillations from bandpass $\Sigma \Delta$ modulators," in Proc. IEEE Int. Symp. on Circuits and Systems, Atlanta, GA, 1996, pp. 469-472.

[37] G. P. Petkov and A. C. Davies, "Constraints on constant-input oscillations of a bandpass sigma-delta modulator structure," Int. J. Circuit Theory Appl., vol. 25, pp. 393-405, 1997.

[38] P. Steiner and W. Yang, "A framework for analysis of high-order sigma-delta modulators," IEEE Trans. Circuits Syst. II, Analog Digit. Signal Process., vol. 44, no. 1, pp. 1-10, Jan. 1997.

[39] N. Wong and T.-S. Ng, "DC stability analysis of high-order, low-pass $\Sigma \Delta$ modulators with distinct unit circle NTF zeros," IEEE Trans. Circuits Syst. II, Analog Digit. Signal Process., vol. 50, no. 1, pp. 12-29, Jan. 2003.

[40] G. Luckjiff and I. Dobson, "Hexagonal sigma-delta modulation," IEEE Trans Circuits Syst. I, Fundam. Theory Appl., vol. 50, no. 8, pp. 991-1005, Aug. 2003.

[41] G. I. Bourdopoulos and T. L. Deliyannis, "High-order vector sigma-delta modulators," IEEE Trans. Circuits Syst. II, Analog Digit. Signal Process., vol. 47, no. 6, pp. 493-503, Jun. 2000.

[42] P. W. Wong, "Fully sigma-delta modulation encoded FIR filters," IEEE Trans. Signal Processing, vol. 40, no. 6, pp. 1605-1610, Jun. 1992.

[43] S. R. Powell and P. M. Chau, "Efficient narrowband FIR and IFIR filters based on powers-of-two sigma-delta coefficient truncation," IEEE Trans. Circuits Syst. II, Analog Digit. Signal Process., vol. 41, no. 8, pp. 497-505, Aug. 1994.

[44] L. Paramesh and A. von Jouanne, "Use of sigma-delta modulation to control EMI from switch-mode power supplies," IEEE Trans. Ind. Electron., vol. 48, no. 1, pp. 111-117, Feb. 2001.
[45] T. Yamanaka, R. Matsumoto, and T. Nakamoto, "Study of odor blender using solenoid valves controlled by delta-sigma modulation method for odor recorder," Sensors Actuators B, vol. 87, pp. 457-463, 2002.

[46] O. Feely and L. O. Chua, "The effect of integrator leak in $\Sigma-\Delta$ modulation,” IEEE Trans. Circuits Syst., vol. 38, no. 11, pp. 1293-1305, Nov. 1991.

[47] S. J. Park and R. M. Gray, "Sigma-delta modulation with leaky integration and constant input," IEEE Trans. Inform. Theory, vol. 38, no. 5, pp. 1512-1533, Sep. 1992.

[48] C. K. Ong, J. L. Huang, and K. T. Cheng, "Testing second-order delta-sigma modulators using pseudo-random patterns," Microelectron. J., vol. 33, pp. 807-814, 2002.

[49] P. Malcovati, S. Brigati, F. Francesconi, F. Maloberti, P. Cusinato, and A. Baschirotto, "Behavioral modeling of switched-capacitor sigma-delta modulators," IEEE Trans Circuits Syst. I, Fundam. Theory Appl., vol. 50, no. 3, pp. 352-364, Mar. 2003.

[50] R. M. Gray, "Spectral analysis of quantization noise in a single-loop sigma-delta modulator with dc input," IEEE Trans. Commun., vol. 37, no. 6, pp. 588-599, Jun. 1989.

[51] J. C. Kieffer, "Analysis of dc input response for a class of one-bit feedback encoders," IEEE Trans. Commun., vol. 38, no. 3, pp. 337-340, Mar. 1990.

[52] D. F. Delchamps, "Nonlinear dynamics of oversampling A-to-D converters," in Proc. IEEE Conf. Decision Control, 1993, pp. 480-485.

[53] R. Schreier, M. V. Goodson, and B. Zhang, "An algorithm for computing convex positively invariant sets for delta-sigma modulators," IEEE Trans Circuits Syst. I, Fundam. Theory Appl., vol. 44, no. 1, pp. 38-44, Jan. 1997.

[54] W. Chou and R. M. Gray, "Dithering and its effects on sigma-delta and multistage sigma-delta modulation," IEEE Trans. Inform. Theory, vol. 37, no. 3, pp. 500-513, May 1991.

[55] R. M. Gray and T. G. Stockham, "Dithered quantizers," IEEE Trans. Inform. Theory, vol. 39, no. 3, pp. 805-812, May 1993.

[56] R. A. Wannamaker, S. P. Lipshitz, J. Vanderkooy, and J. N. Wright, "A theory of nonsubtractive dither," IEEE Trans. Signal Processing, vol. 48, no. 2, pp. 499-516, Feb. 2000.

[57] P. J. Ramadge, "On the periodicity of symbolic observations of piecewise-smooth discrete-time systems," IEEE Trans. Automat. Contr., vol. 35, no. 7, pp. 807-813, Jul. 1990.

[58] C. W. Wu and L. O. Chua, "Symbolic dynamics of piecewise-linear maps," IEEE Trans. Circuits Syst. II, Analog Digit. Signal Process., vol. 41, no. 6, pp. 420-424, Jun. 1994.

[59] A. Kirac and P. P. Vaidyanathan, "Results on lattice vector quantization with dithering," IEEE Trans. Circuits Syst. II, Analog Digit. Signal Process., vol. 43, no. 12, pp. 811-825, Dec. 1996.

[60] S. M. Moussavi and B. H. Leung, "High-order single-stage single-bit oversampling A/D converter stabilized with local feedback loops," IEEE Trans. Circuits Syst. II, Analog Digit. Signal Process., vol. 41, no. 1, pp. 19-25, Jan. 1994.

[61] A. Pneumatikakis, A. G. Constantinides, T. Deliyannis, and G. Bourdopoulos, "Stabilization of third-order, single-stage, sigma-delta modulators," Circuits, Syst. Signal Proces., vol. 18, no. 2, pp. 149-168, 1999.

[62] A. J. Magrath and M. B. Sandler, "Efficient dithering of sigma-delta modulators with adaptive bit flipping," Electron. Lett., vol. 31, no. 11, pp. 846-947, 1995.

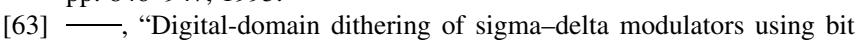
flipping," J. Audio Eng. Soc., vol. 45, no. 6, pp. 467-475, 1997.

[64] X. Sun and K. R. Laker, "Tonal behavior analysis of an adaptive secondorder Sigma-Delta modulator," in Proc. IEEE Int. Symp. Circuits and Systems, vol. 4, 2002, pp. 277-280.

[65] L. Risbo, "Improved stability and performance from sigma-delta modulators using 1-bit vector quantization," in Proc. IEEE Int. Symp. Circuits and Systems, 1993, pp. 1365-1368.

[66] J. L. Willems, Stability Theory of Dynamical Systems. New York: Wiley, 1970.

[67] D. F. Delchamps, "Quantization noise in sigma-delta modulators driven by deterministic inputs," in Proc. Int. Conf. Acoustics, Speech, Signal Processing, vol. 4, San Francisco, CA, Mar. 1992, pp. 425-428.

[68] R. M. Gray, "Quantization noise spectra," IEEE Trans. Inform. Theory, vol. 36, no. 6, pp. 1220-1244, Nov. 1990.

[69] R. E. Kalman and J. E. Bertram, "Control system analysis and design via the "second method" of Lyapunov, Parts I and II," J. Basic Eng., vol. 82, pp. 371-400, 1960. 


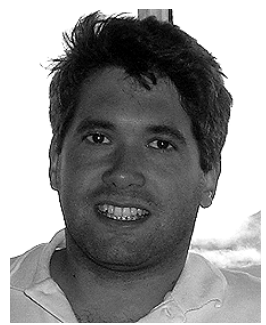

Daniel E. Quevedo (S'97) received the Ingeniero Civil Electrónico and Magister en Ingeniería Electrónica degrees from the Universidad Técnica Federico Santa María, Valparaíso, Chile, in 2000. $\mathrm{He}$ is working toward the Ph.D degree at the School of Electrical Engineering and Computer Science, The University of Newcastle, Newcastle, NSW, Australia, since 2001.

He has lectured on several courses related to automatic control at the Universidad Técnica Federico Santa María and The University of Newcastle. He also has working experience at VEW Energie AG, Dortmund, Germany and at the Cerro Tololo Inter-American Observatory, La Serena, Chile. His main research interests cover several areas of automatic control, signal processing, and communications.

Mr. Quevedo was supported by a full scholarship from the alumni association during his time at the Universidad Técnica Federico Santa María and received several university-wide prizes upon graduating. He received the IEEE Conference on Decision and Control Best Student-Paper Award in 2003 and was also a finalist in 2002. In 2003, he was awarded the University of Newcastle Faculty of Engineering and Built Environment Prize for the Best International Conference Paper.

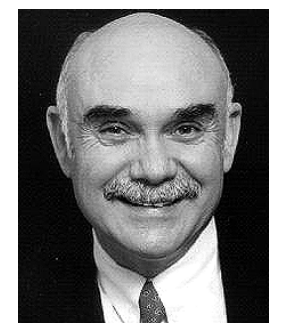

Graham C. Goodwin (M'74-SM'84-F'86) received the B.Sc. degree in physics, the B.E. degree in electrical engineering, and the $\mathrm{Ph} . \mathrm{D}$ degree from the University of New South Wales, Sydney, Australia.

From 1970 until 1974, he was a Lecturer in the Department of Computing and Control, Imperial College, London, U.K. Since 1974, he has been with the Department of Electrical and Computer Engineering, The University of Newcastle, Newcastle, NSW, Australia. He is currently Professor of Electrical Engineering, Associate Director of the Centre for Complex Dynamic Systems and Control at the University of Newcastle, and a Director of National ICT, Australia. He is the co-author of eight monographs: Control Theory (Edinburgh, U.K.: Oliver and Boyd, 1970), Dynamic System Identification (New York, Academic, 1977), Adaptive Filtering, Prediction and Control (Englewood Cliffs, NJ: Prentice-Hall, 1984), Digital Control and Estimation (Englewood Cliffs, NJ: Prentice-Hall, 1989), Sampling in Digital Signal Processing and Control (Cambridge, MA: Birkhauser, 1996), Fundamental Limitations in Filtering and Control (New York, Springer, 1997), Control System Design (Englewood Cliffs, NJ: Prentice-Hall, 2001), Constrained Control and Estimation (New York, Springer, 2004); four edited volumes, and several hundred technical papers.

Dr. Goodwin is the recipient of several international prizes including the USA Control Systems Society 1999 Hendrik Bode Lecture Prize, a Best Paper award by IEEE TRANSACTIONS ON AUTOMATIC CONTROL, a Best Paper award by Asian Journal of Control, and Best Engineering Text Book award from the International Federation of Automatic Control. He is the recipient of an ARC Federation Fellowship; an Honorary Fellow of the Institute of Engineers, Australia; a Fellow of the Australian Academy of Science; a Fellow of the Australian Academy of Technology, Science and Engineering; a Member of the International Statistical Institute; a Fellow of the Royal Society, London, and a Foreign Member of the Royal Swedish Academy of Sciences. 